\title{
CONNECTING SUBSTRUCTURE IN GALAXY CLUSTER CORES AT $z=0.2$ WITH CLUSTER ASSEMBLY HISTORIES
}

\author{
GRAHAM P. SMith ${ }^{1,2}$ AND JAMES E. TAYLOR ${ }^{2,3}$ \\ Received 2008 April 25; accepted 2008 June 24; published 2008 July 8
}

\begin{abstract}
We use semianalytic models of structure formation to interpret gravitational lensing measurements of substructure in galaxy cluster cores $\left(R \leq 250 h^{-1} \mathrm{kpc}\right)$ at $z=0.2$. The dynamic range of the lensing-based substructure fraction measurements is well matched to the theoretical predictions, both spanning $f_{\text {sub }} \sim 0.05-0.65$. The structure formation model predicts that $f_{\text {sub }}$ is correlated with cluster assembly history. We use simple fitting formulae to parameterize the predicted correlations: $\Delta_{90}=\tau_{90}+\alpha_{90} \log \left(f_{\text {sub }}\right)$ and $\Delta_{50}=\tau_{50}+\alpha_{50} \log \left(f_{\text {sub }}\right)$, where $\Delta_{90}$ and $\Delta_{50}$ are the predicted lookback times from $z=0.2$ to when each theoretical cluster had acquired $90 \%$ and $50 \%$, respectively, of the mass it had at $z=0.2$. The best-fit parameter values are $\alpha_{90}=-1.34 \pm 0.79 \mathrm{Gyr}, \tau_{90}=$ $0.31 \pm 0.56 \mathrm{Gyr}$ and $\alpha_{50}=-2.77 \pm 1.66 \mathrm{Gyr}, \tau_{50}=0.99 \pm 1.18 \mathrm{Gyr}$. Therefore, (1) observed clusters with $f_{\text {sub }} \lesssim 0.1$ (e.g., A383, A1835) are interpreted, on average, to have formed at $z \gtrless 0.8$ and to have suffered $\leq 10 \%$ mass growth since $z \simeq 0.4$, and (2) observed clusters with $f_{\text {sub }} \geqslant 0.4$ (e.g., A68, A773) are interpreted as, on average, forming since $z \simeq 0.4$ and suffering $>10 \%$ mass growth in the $\sim 500 \mathrm{Myr}$ preceding $z=0.2$, i.e., since $z=0.25$. In summary, observational measurements of $f_{\text {sub }}$ can be combined with structure formation models to estimate the age and assembly history of observed clusters. The ability to "age date" approximately clusters in this way has numerous applications to the large samples of clusters that are becoming available.
\end{abstract}

Subject headings: cosmology: observations - cosmology: theory - galaxies: clusters: general galaxies: clusters: individual (A68, A209, A267, A383, A773, A963, A1763, A1835, A2218, A2219) — gravitational lensing

Online material: color figure

\section{INTRODUCTION}

The mass growth of clusters is sensitive to the dark energy equation-of-state parameter $w$, the matter density of the universe $\Omega_{M}$, and the normalization of the matter power spectrum $\sigma_{8}$ (e.g., Evrard et al. 1993; Smith et al. 2003; Mantz et al. 2008). Clusters are inferred to grow hierarchically via the ingestion of smaller dark matter halos (that host galaxies) into the more massive parent halo (cluster). The structure of galaxy clusters, specifically the internal substructure of clusters, therefore contains a wealth of cosmological information, including possible clues about the physics of the dark matter particle itself (e.g., Natarajan et al. 2002). From an astrophysical point of view, the mass growth of clusters brings new (generally gasrich) galaxy populations into clusters (e.g., Moran et al. 2007) and may lead to shock heating of the intracluster medium and/ or disruption of cooling in cluster cores (e.g., Poole et al. 2008). Reliable measurement and interpretation of cluster substructure are therefore of broad interest.

The most direct way to detect substructure within clusters is via gravitational lensing. Group-scale substructures within individual clusters were detected in early ground-based stronglensing studies of individual clusters (Pello et al. 1991; Kneib et al. 1993, 1995) and subsequently measured to high precision using Hubble Space Telescope (HST) data (Kneib et al. 1996). Smith et al. (2005, hereafter Sm05; see § 2) then measured the structure of a sample of 10 clusters at $z \simeq 0.2$. In this Letter we use Taylor \& Babul's (2004, hereafter TB04) semianalytical

\footnotetext{
${ }^{1}$ School of Physics and Astronomy, University of Birmingham, Edgbaston, Birmingham, B15 2TT, UK; gps@star.sr.bham.ac.uk.

${ }^{2}$ California Institute of Technology, Mail Code 105-24, 1200 East California Boulevard, Pasadena, CA 91125.

${ }^{3}$ Department of Physics and Astronomy, University of Waterloo, Waterloo, ON N2L 3G1, Canada; taylor@sciborg.uwaterloo.ca.
}

models of structure formation to interpret Sm05's cluster substructure measurements, as a means of exploring lensing-based substructure measurements as a quantitative probe of cluster age and assembly history.

We summarize Sm05 and TB04 in $\S 2$ and $\S 3$, respectively, and then synthesize observations and theory in $\S 4$. We discuss caveats in $\S 5$ and summarize our conclusions and discuss future prospects in $\S 6$. We assume $H_{0}=70 \mathrm{~km} \mathrm{~s}^{-1} \mathrm{Mpc}^{-1}$, $\Omega_{M}=0.3, \Omega_{\Lambda}=0.7$, and $\sigma_{8}=0.9$ throughout. The lookback time from $z=0$ to $z=0.2$ is $t_{z=0.2}=2.44 \mathrm{Gyr}$ in this cosmology.

\section{SUMMARY OF OBSERVATIONAL RESULTS}

Sm05 investigated the projected mass and structure of 10 X-ray-luminous $\left(L_{\mathrm{X}} \geq 4 \times 10^{44} \mathrm{ergs} \mathrm{s}^{-1}, 0.1-2.4 \mathrm{keV}\right)$ cluster cores at $0.17 \leq z \leq 0.25$ (Table 1). HST/WFPC2 imaging and ground-based spectroscopy of gravitational arcs (Smith et al. 2001, 2002; Sand et al. 2005; Richard et al. 2007), were used to characterize the strong and weak gravitational lensing signal of each cluster core. The lensing signals were then used to constrain a detailed parametrized model of the projected mass distribution in each cluster core following Kneib (1993; see also Kneib et al. 1996 and Smith 2002). Each lens model includes mass components that account for both the underlying dark matter distribution in the cluster (cluster/group-scale mass components) and the cluster galaxies down to $L_{\mathrm{K}} \geq 0.1 L_{\mathrm{K}}^{*}$. For clarity, we refer to the main central cluster dark matter halo as the cluster-scale mass component, and all other massive substructures associated with infallen clusters and/or groups as group-scale mass components. The typical number of galaxyscale mass components in each lens model was 30 .

The HST data probe out to a typical projected clustercentric radius of $R=250 h^{-1} \mathrm{kpc}$ at the cluster redshifts. Sm05 mea- 
TABLE 1

Observational Substructure Measurements

\begin{tabular}{|c|c|c|c|c|c|c|c|c|}
\hline Cluster & Redshift & $\begin{array}{c}M_{\mathrm{tot}} \\
\left(10^{14} M_{\odot}\right)\end{array}$ & $N_{\text {DM }}$ & $f_{\text {sub }}$ & Strong Lensing? & Sm05 Classification & $\begin{array}{c}\Delta_{50} \\
(\mathrm{Gyr})\end{array}$ & $\begin{array}{c}\Delta_{90} \\
(\mathrm{Gyr})\end{array}$ \\
\hline A383 & 0.188 & $2.6 \pm 0.1$ & 1 & $0.06 \pm 0.01$ & Confirmed & Undisturbed unimodal & $4.3 \pm 2.4$ & $2.0 \pm 1.1$ \\
\hline A963 & 0.206 & $2.4 \pm 0.2$ & 1 & $0.06 \pm 0.01$ & Confirmed & Undisturbed unimodal & $4.3 \pm 2.4$ & $2.0 \pm 1.1$ \\
\hline A1835 & 0.253 & $4.1 \pm 1.1$ & 1 & $0.06 \pm 0.01$ & Unconfirmed & Undisturbed unimodal & $4.3 \pm 2.4$ & $2.0 \pm 1.1$ \\
\hline A267 & 0.230 & $1.9 \pm 0.4$ & 1 & $0.08 \pm 0.01$ & Unconfirmed & Disturbed unimodal & $4.0 \pm 2.2$ & $1.8 \pm 1.0$ \\
\hline A 1763 & 0.288 & $1.5 \pm 0.8$ & 1 & $0.15 \pm 0.05$ & No? & Disturbed unimodal & $3.2 \pm 1.8$ & $1.4 \pm 0.9$ \\
\hline A209 & 0.209 & $1.1 \pm 0.5$ & 1 & $0.18 \pm 0.06$ & No? & Disturbed unimodal & $3.0 \pm 1.7$ & $1.3 \pm 0.8$ \\
\hline A2219 & 0.228 & $2.4 \pm 0.1$ & 3 & $0.20 \pm 0.01$ & Confirmed & Disturbed multimodal & $2.9 \pm 1.7$ & $1.3 \pm 0.8$ \\
\hline A2218 & 0.171 & $4.0 \pm 0.1$ & 2 & $0.27 \pm 0.01$ & Confirmed & Disturbed multimodal & $2.5 \pm 1.5$ & $1.1 \pm 0.7$ \\
\hline A68 ......... & 0.255 & $3.1 \pm 0.1$ & 2 & $0.36 \pm 0.01$ & Confirmed & Disturbed multimodal & $2.2 \pm 1.4$ & $0.9 \pm 0.7$ \\
\hline A773 & 0.217 & $3.6 \pm 1.2$ & 3 & $0.61 \pm 0.20$ & Unconfirmed & Disturbed multimodal & $1.6 \pm 1.3$ & $0.6 \pm 0.6$ \\
\hline
\end{tabular}

sured the cluster substructure within this radius. Here we define $f_{\text {sub }}$ as the fraction of mass associated with substructures, $f_{\text {sub }} \equiv M_{\text {sub }} / M_{\text {tot }}=1-M_{\text {cen }} / M_{\text {tot }}$, where $M_{\text {cen }} / M_{\text {tot }}$ is the central mass fraction used by Sm05. For this purpose, "substructures" include group-scale masses and galaxy-scale masses, with the exception of the brightest cluster galaxy (BCG). This is because BCG mass is degenerate with cluster-scale mass in the lens models, and, in any event, Sm05 found no evidence for BCGs being offset from the center of the cluster-scale mass components. The clusters are listed in order of increasing $f_{\text {sub }}$ in Table 1 , together with $N_{\mathrm{DM}}$, the number of cluster/group-scale mass components in each lens model. As expected, $N_{\text {DM }}$ and $f_{\text {sub }}$ are correlated; however, there is a factor of 3 spread in $f_{\text {sub }}$ for subsamples of clusters with $N_{\mathrm{DM}}=1$ and $N_{\mathrm{DM}}>1$. Note that to aid comparison of Sm05's lens modeling results with predictions, the values of $f_{\text {sub }}$ in Table 1 have been adjusted upward to take account of the fact that the L-shaped observed WFPC2 field of view $\left(\sim 5 \operatorname{arcmin}^{2}\right)$ covers just $\sim 50 \%$ of a circle of radius $250 h^{-1} \mathrm{kpc}\left(\sim 10 \operatorname{arcmin}^{2}\right)$. The WFPC2 observations were originally designed to include the likely group-scale substructures in the cluster cores. The adjustments to $f_{\text {sub }}$ therefore account statistically for the galaxy-scale masses not included in Sm05's analysis, leaving $N_{\text {DM }}$ unchanged.

Cluster-to-cluster differences in $f_{\text {sub }}$ arise for two reasons: (1) group-scale mass components in the lens models that are associated with massive, likely infalling structures such as groups of galaxies and (2) cluster galaxies that are associated both with the central cluster dark matter halo (and are presumably virialized) and with the infalling structures. Sm05 broadly interpreted measurements of $f_{\text {sub }}$ as indicating the merger history of clusters; however, quantitative conclusions on cluster assembly and age were impossible without theoretical models.

\section{SUMMARY OF THEORETICAL MODELS}

TB04's semianalytic model of halo evolution provides a fast way of generating a large number of model halos for comparison with observations and agrees well in its predictions with self-consistent $n$-body simulations (Taylor \& Babul 2005). The model includes two main components: a merger-tree code for determining the assembly history of an individual cluster and an analytic description of how the main halo and the merging subcomponent evolve after each merger. We will summarize these components here and refer the reader to TB04 for full details.

The merger tree describing the assembly of a single cluster is generated randomly using the algorithm of Somerville \& Kolatt (1999). Starting from a halo of specified mass at $z=0$, the algorithm chooses an interval to step back in redshift and picks progenitors at this redshift following extended Press-
Schechter merger statistics (Lacey \& Cole 1993). Iterating produces a complete history of the mergers through which the final object assembled, down to some limiting mass resolution and back to some redshift. In this Letter we use a set of 1000 merger trees whose final masses at $z=0$ are randomly drawn from the massive end $\left(>5 \times 10^{14} M_{\odot}\right)$ of a halo mass function. For the range of concentration parameters derived for our halos (as explained below), this produces projected masses within a clustercentric radius of $R=250 h^{-1} \mathrm{kpc}$ (integrating out to the virial radius along the line of sight) in the range $(0.4-8) \times$ $10^{14} M_{\odot}$, similar to those in the observed sample (Table 1). The merger trees for these systems are followed back to a redshift of 50, or until they drop below the resolution limit of $10^{-4}$ times the final mass.

Given a merger tree, the model of TB04 selects the most massive progenitor in the most recent merger and traces its history back, selecting the most massive progenitor at each time step. This object is considered the "main" system and is modeled as a spherical halo with a radial density profile similar to the "universal" profile found in simulations (Navarro et al. 1997). To account for some of the centrally concentrated (baryonic) mass in the cluster, we use the fitting formula proposed by Moore et al. (1998), which has a steeper $r^{-1.5}$ central cusp. In an update to the model presented in TB04, in this work we use the whole mass assembly history of the system, rather than just its instantaneous mass, to derive the value of its concentration parameter at each redshift step, following Wechsler et al. (2002). Each merging subhalo is added to this main system as a smaller spherical object with a Moore profile, and its subsequent orbit and mass-loss history are determined using the analytic description of dynamical friction, tidal stripping, and tidal heating in Taylor \& Babul (2001). Subhalos are tracked until they are disrupted either by repeated tidal stripping or by passing within $0.01 R_{\mathrm{vir}}$ of the center of the main potential. The model also includes simple treatments of subsubstructure (subhalos within subhalos), correlated orbits in infalling groups of objects, and collisions and encounters between objects. See TB04 for a detailed explanation of these elements.

For each merger tree, we use the semianalytic model to determine how much substructure exists at $z=0.2$ and calculate $f_{\text {sub }}$ as the fraction of the mass projected within $250 \mathrm{~h}^{-1}$ $\mathrm{kpc}$ of the halo center that is contained in the 30 most massive subhalos in the system (to match the observations; see $\S 2$ ). We exclude from the final analysis any substructure that ends up within $5 \%$ of $R_{\text {vir }}$ of the halo center, since in a real cluster most such objects would interact strongly and merge rapidly with the dominant central galaxy. We also record several indicators of the mass assembly history of the main system in each merger tree, including $t_{90}$ and $t_{50}$, the lookback times by 
which it had assembled $90 \%$ and $50 \%$, respectively, of the mass it has at redshift 0.2 . These thresholds are chosen to probe the recent infall history and cluster age, respectively. We show the resulting $f_{\text {sub }}-t_{90}$ and $f_{\text {sub }}-t_{50}$ distributions in Figure 1. The observed clusters span a dynamic range of $0.06 \leq f_{\text {sub }} \leq 0.62$ (Table 1), which is comparable with that of the TB04 theoretical models and consistent with other theoretical estimates of cluster substructure (e.g., Diemand et al. 2004; Gao et al. 2004; Nagai \& Kravtsov 2005; van den Bosch et al. 2005).

\section{RESULTS}

Figure 1 reveals that the TB04 model predicts that $f_{\text {sub }}$ and $t_{90}$ are correlated, as are $f_{\text {sub }}$ and $t_{50}$. However, at $f_{\text {sub }}<0.2$ the distributions of $t_{90}$ and $t_{50}$ are bimodal-some theoretical clusters exhibiting both a low substructure fraction and recent significant infall. The unphysical location of these clusters in Figure 1 is due to a timing difference between the axes $-t_{90}$ is sensitive to mass growth within the virialized region of the cluster (approximately a sphere of diameter $\sim 3 \mathrm{Mpc}$ ); $f_{\text {sub }}$ is sensitive to substructures in a cylinder of diameter $500 \mathrm{~h}^{-1} \mathrm{kpc}$ through the center of the cluster. If an infalling structure has crossed the virial radius but not yet entered the cylinder, then it may appear in the "spike" of clusters at $f_{\text {sub }}<0.2$ and $t_{90}<$ $3 \mathrm{Gyr}$ (or $t_{50}<3 \mathrm{Gyr}$ ). We exclude these clusters from the calculations described below.

We have therefore confirmed and considerably extended Sm05's qualitative interpretation of the lensing results: clusters with high substructure fractions have (1) suffered more pronounced recent infall than clusters with low substructure fractions and (2) formed at later times than clusters with lower substructure fractions. To quantify this, we fit the following simple formulae to the theoretical data: $\Delta_{90}=t_{90}-$ $t_{z=0.2}=\tau_{90}+\alpha_{90} \log \left(f_{\text {sub }}\right)$ and $\Delta_{50}=t_{50}-t_{z=0.2}=\tau_{50}+$ $\alpha_{50} \log \left(f_{\text {sub }}\right)$, where $\alpha_{90}$ and $\alpha_{50}$ parameterize the dependence of $\Delta_{90}$ and $\Delta_{50}$, respectively, on $f_{\text {sub }} ; \tau_{90}$ and $\tau_{50}$ are the intercepts at $f_{\text {sub }}=1$. We first bin up the individual theoretical data points in bins of width $\Delta \log \left(f_{\text {sub }}\right)=0.2$ and then weight each bin in the fit by the reciprocal of the sample variance in the respective bin. The best-fit parameter values obtained in this way are $\alpha_{90}=-1.34 \pm 0.79 \mathrm{Gyr}, \tau_{90}=0.31 \pm$ $0.56 \mathrm{Gyr}$ and $\alpha_{50}=-2.77 \pm 1.66 \mathrm{Gyr}, \tau_{50}=0.99 \pm 1.18$ Gyr. The $\tau_{90}$ and $\tau_{50}$ parameters are measurements of the timing difference between the axes in Figure 1 discussed above and are well matched to the infall timescale of $\sim 0.5-1$ Gyr.

The best-fit models were then used to interpret quantitatively the observed substructure fractions from Sm05 listed in Table 1 -i.e., to estimate the age $\left(\Delta_{50}\right)$ and recent infall history $\left(\Delta_{90}\right)$ of each cluster (see Table 1). The uncertainties on $\Delta_{90}$ and $\Delta_{50}$ quoted in Table 1 incorporate errors on $f_{\text {sub }}$ and on the best-fit parameter values derived above and are dominated by the scatter on $f_{\text {sub }}-t_{50}$ and $f_{\text {sub }}-t_{90}$. The typical uncertainty on cluster age is $\sim 1.7 \mathrm{Gyr}$, with ages spanning $\sim 1-4 \mathrm{Gyr}$. Clusters with the largest substructure fractions, i.e., $f_{\text {sub }} \geq 0.4$, formed within the $\sim 2$ Gyr preceding $z=0.2$, i.e., since $z \lesssim 0.4$, and had not assembled $90 \%$ of the mass they had at $z=0.2$ until $z \simeq 0.25$, i.e., just $\sim 500 \mathrm{Myr}$ before $z=0.2$. In contrast, clusters with the lowest substructure fractions, i.e., $f_{\text {sub }}<0.1$, formed $\geq 4 \mathrm{Gyr}$ before $z=0.2$, i.e., at $z \geq 0.8$, and then went on to assemble $>90 \%$ of their $z=0.2$ mass within the ensuing $\sim 2 \mathrm{Gyr}$ and suffered negligible mass growth in the $\sim 2 \mathrm{Gyr}$ prior to $z=0.2$, i.e., since $z \simeq 0.4$. Therefore we interpret clusters with the lowest $f_{\text {sub }}$ as being, on average, almost fully

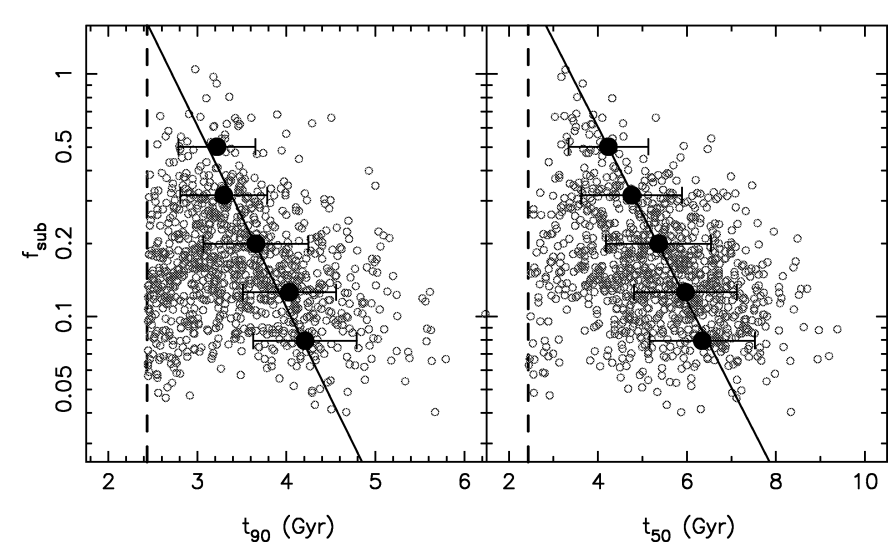

FIG. 1.-Predicted distribution of substructure fraction vs. $t_{90}$ (left) and $t_{50}$ (right). Note that the lookback time from $z=0$ to $z=0.2$ is $2.4 \mathrm{Gyr}$ (dashed line). The gray open points show clusters from TB04's model-they span a similar dynamic range to the observed clusters (see Table 1). The large black points show the mean prediction in equally spaced logarithmic bins; the error bars show the $1 \sigma$ scatter in each bin. The solid black line shows the best-fit relations described in the text $(\S 4)$.

( $\sim 90 \%)$ assembled by the time that the clusters with highest $f_{\text {sub }}$ had barely formed (i.e., assembled half of their mass).

\section{CAVEATS}

TB04 make some simplifying assumptions that may affect our results: (1) dark matter halos are assumed to be spherical, whereas real dark matter halos are likely triaxial; (2) all matter is treated as collisionless, thus ignoring baryonic physics including adiabatic contraction due to gas cooling and the dynamical effects of galaxies; and (3) matter outside the cluster virial radius is ignored; in contrast, lensing is sensitive to all the matter along the line of sight, including chance foreground and background projections. We will consider these complications in more detail in future work.

The main systematic uncertainty on the observed substructure fractions is the completeness of Sm05's lens models as a function of subhalo mass. A detailed study of this issue will be published in the future (V. Hamilton-Morris et al., in preparation). Here we identify how many of Sm05's models may suffer from incompleteness. Incompleteness is most likely to arise from features in the dark matter distribution not identified in the luminous properties of the clusters within $R<250 h^{-1}$ $\mathrm{kpc}$, including group-scale dark matter halos in which the cluster galaxies may be embedded. Strong lensing has been detected in eight of the 10 observed clusters (Table 1); group-scale dark matter halos are detected in three of the eight by the measurable way they alter the appearance of strongly lensed arcs compared to simpler mass distributions. Clusters in which strong-lensing has not been detected are therefore of greatest concern, as pointed out in Sm05's discussion of the filament feeding A1763; see also Mercurio et al.'s (2003) discussion of the complex dynamical structure of A209. Gross substructure incompleteness may therefore be a concern for $\sim 20 \%$ of the observed sample.

We also consider whether there is any evidence in the respective $f_{\text {sub }}$ distributions for systematic differences between Sm05's X-ray-selected sample and TB04's mass-selected sample. To address this we compare the cumulative and differential $f_{\text {sub }}$ distributions in Figure 2. A K-S test obtains a probability of $20 \%$ that the observed and theoretical distributions are drawn from the same parent distribution, i.e., formally evidence of systematic differences between the samples at $1.3 \sigma$ signific- 

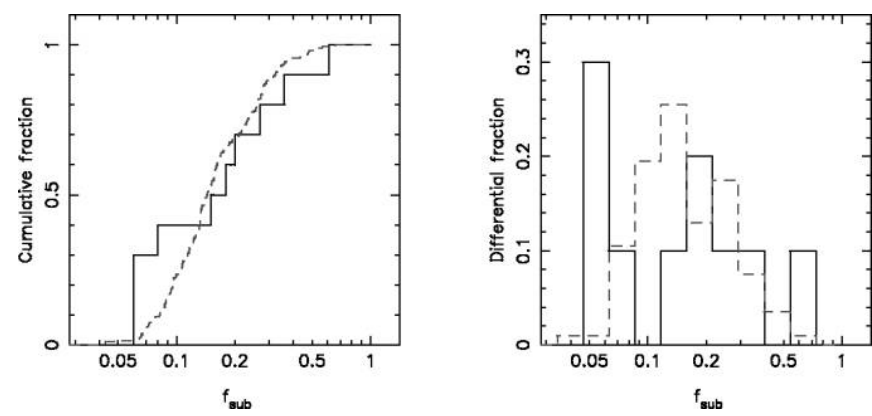

FIG. 2.-Distribution of substructure fractions from the observed sample (black solid line) and from the theoretical model (red dashed line). The observed clusters show a possible excess of very low and very high substructure systems-this is discussed in $\S 5$. [See the electronic edition of the Journal for a color version of this figure.]

ance. The small observed sample size of just 10 clusters is clearly a limiting factor here. The right panel of Figure 2 helps somewhat, as it highlights more clearly the possible bias of the observed sample to very high and very low values of $f_{\text {sub }}$. Such a bias would be plausible because cluster-cluster mergers and cool cores both suffer an excess of X-ray flux at a fixed mass over a nonmerging, noncool core cluster. A much larger observational sample is required to investigate this issue further.

\section{CONCLUSIONS AND FUTURE PROSPECTS}

We have combined theoretical models of structure formation (TB04) with gravitational lens models of galaxy clusters (Sm05) to explore how measurements of cluster substructure from lensing observations can be interpreted in the context of the age and assembly history of clusters. The main result is that $f_{\text {sub }}$, the fraction of cluster mass within a projected clustercentric radius of $R=250 \mathrm{~h}^{-1} \mathrm{kpc}$ associated with substructure (galaxies and group-scale halos), as can be measured from lensing data, is predicted to be strongly correlated with the age and recent mass growth of galaxy clusters. We fitted the following simple formulae to the theoretical data to quantify the predicted behavior in a convenient form: $\Delta_{90}=t_{90}-$ $t_{z=0.2}=\tau_{90}+\alpha_{90} \log \left(f_{\text {sub }}\right) \quad$ and $\quad \Delta_{50}=t_{90}-t_{z=0.2}=\tau_{50}+$ $\alpha_{50} \log \left(f_{\text {sub }}\right)$, where $t_{90}$ and $t_{50}$ are the lookback times at which a cluster had acquired $90 \%$ and $50 \%$ of its mass at $z=0.2$. The best-fit parameter values are $\alpha_{90}=-1.34 \pm 0.79 \mathrm{Gyr}$, $\tau_{90}=0.31 \pm 0.56 \mathrm{Gyr}$ and $\alpha_{50}=-2.77 \pm 1.66 \mathrm{Gyr}, \tau_{50}=$ $0.99 \pm 1.18$ Gyr. Low- $f_{\text {sub }}$ clusters $\left(f_{\text {sub }} \lesssim 0.1\right.$; e.g., A383, A1835) are therefore interpreted as, on average, having formed at $z \gtrsim 0.8$ and having suffered $\leq 10 \%$ mass growth in the 2 Gyr preceding $z=0.2$, i.e., since $z \simeq 0.4$. In contrast, high$f_{\text {sub }}$ clusters $\left(f_{\text {sub }} \geqslant 0.4\right.$; e.g., A68, A773) are interpreted, on average, to have formed just $\sim 2 \mathrm{Gyr}$ before $z=0.2$, i.e., since $z \simeq 0.4$, and suffered $10 \%$ mass growth in the $\sim 0.5 \mathrm{Gyr}$ preceding $z=0.2$, i.e., since $z \simeq 0.25$.

Our synthesis therefore demonstrates that lensing-based measurements of $f_{\text {sub }}$ can be combined with semianalytic structure formation models to estimate the average age and assembly history of observed clusters. This suggests numerous avenues for further exploration, including (1) expansion of the observed samples by at least an order of magnitude; (2) calibration of the completeness of the lensing-based mass function of subhalos in clusters; (3) investigation of how lensing-based cluster age and assembly history estimates might allow new cosmological constraints to be derived, for example, on the dark energy equation-of-state parameter $w$; and (4) analysis of cluster galaxy populations and cluster scaling relations as a function of cluster age.

We thank Arif Babul, Habib Khosroshahi, Jean-Paul Kneib, Ian Smail, and Risa Wechsler for helpful discussions. G. P. S. acknowledges support from NASA (HST-GO-10420.04-A), Caltech, and a Royal Society University Research Fellowship. J. E. T. acknowledges financial support from the US NSF (grant AST-0307859) and DoE (contract DE-FG02-04ER41316) and from NSERC Canada.

\section{REFERENCES}

Diemand, J., Moore, B., \& Stadel, J. 2004, MNRAS, 352, 535

Evrard, A. E., Mohr, J. J., Fabricant, D. G., \& Geller, M. J. 1993, ApJ, 419, L9

Gao, L., White, S. D. M., Jenkins, A., Stoehr, F., \& Springel, V. 2004, MNRAS, 355,819

Kneib, J.-P. 1993, Ph.D. thesis, Univ. Paul Sabatier

Kneib, J.-P., Ellis, R. S., Smail, I., Couch, W. J., \& Sharples, R. M. 1996, ApJ, 471, 643

Kneib, J. P., Mellier, Y., Fort, B., \& Mathez, G. 1993, A\&A, 273, 367

Kneib, J. P., Mellier, Y., Pello, R., Miralda-Escudé, J., Le Borgne, J.-F., Boehringer, H., \& Picat, J.-P. 1995, A\&A, 303, 27

Lacey, C., \& Cole, S. 1993, MNRAS, 262, 627

Mantz, A., Allen, S. W., Ebeling, H., \& Rapetti, D. 2008, MNRAS, 387, 1179

Mercurio, A., Girardi, M., Boschin, W., Merluzzi, P., \& Busarello, G. 2003, A\&A, 397, 431

Moore, B., Governato, F., Quinn, T., Stadel, J., \& Lake, G. 1998, ApJ, 499, L5

Moran, S. M., Ellis, R. S., Treu, T., Smith, G. P., Rich, R. M., \& Smail, I. 2007, ApJ, 671, 1503

Nagai, D., \& Kravtsov, A. V. 2005, ApJ, 618, 557

Natarajan, P., Loeb, A., Kneib, J.-P., \& Smail, I. 2002, ApJ, 580, L17

Navarro, J. F., Frenk, C. S., \& White, S. D. M. 1997, ApJ, 490, 493
Pello, R., Sanahuja, B., Le Borgne, J., Soucail, G., \& Mellier, Y. 1991, ApJ, 366, 405

Poole, G. B., Babul, A., McCarthy, I. G., Sanderson, A. J. R., \& Fardal, M. A. 2008, preprint (arXiv:0804.1552)

Richard, J., et al. 2007, ApJ, 662, 781

Sand, D. J., Treu, T., Ellis, R. S., \& Smith, G. P. 2005, ApJ, 627, 32

Smith, G. P. 2002, Ph.D. thesis, Univ. Durham

Smith, G. P., Edge, A. C., Eke, V. R., Nichol, R. C., Smail, I., \& Kneib, J. 2003, ApJ, 590, L79

Smith, G. P., Kneib, J., Ebeling, H., Czoske, O., \& Smail, I. 2001, ApJ, 552, 493

Smith, G. P., Kneib, J.-P., Smail, I., Mazzotta, P., Ebeling, H., \& Czoske, O. 2005, MNRAS, 359, 417 (Sm05)

Smith, G. P., Smail, I., Kneib, J.-P., Davis, C. J., Takamiya, M., Ebeling, H., \& Czoske, O. 2002, MNRAS, 333, L16

Somerville, R. S., \& Kolatt, T. S. 1999, MNRAS, 305, 1

Taylor, J. E., \& Babul, A. 2001, ApJ, 559, 716

- 2004, MNRAS, 348, 811 (TB04)

2005, MNRAS, 364, 535

van den Bosch, F. C., Tormen, G., \& Giocoli, C. 2005, MNRAS, 359, 1029

Wechsler, R. H., Bullock, J. S., Primack, J. R., Kravtsov, A. V., \& Dekel, A. 2002, ApJ, 568, 52 